\title{
The search for the sub-solar initial mass function in the Local Group
}

\author{
Dimitrios Gouliermis, Alessandro Berton, Wolfgang Brandner, \\ Thomas Henning and Markus Feldt
}

Max-Planck-Institut für Astronomie, Königstuhl 17, D-69117 Heidelberg, Germany email: [dgoulier; berton; brandner; henning; feldt]@mpia.de

\begin{abstract}
The stellar Initial Mass Function (IMF) is a quantity which accounts for the distribution of the masses of stars, when they are formed. All the information available on the IMF in the low-mass regime comes from studies of our galaxy alone. Investigations on the content of low-mass stars in other neighbouring galaxies are limited by observational constraints, which do not allow the detection of the fainter stars with statistical significance. Only recently results from observations with the Hubble Space Telescope (HST) of stellar populations in the Large Magellanic Cloud (LMC) down to $\sim 0.7 \mathrm{M}_{\odot}$ confirm systematic variations in the low-mass IMF expected from theoretical considerations (Gouliermis et al. 2005). Direct imaging of resolved stellar populations in massive young clusters throughout the Local Group would be possible with Extremely Large Telescopes (ELTs). Hence, a sizeable sample of young clusters for which IMF variations can be detected would become available. We present our method for testing the efficiency of observations with ELTs in detecting low-mass stars in compact clusters of the Local Group galaxies. We plan to simulate imaging with ELTs and use the results of their photometry in order to investigate the effect on the derived low-mass IMF. This method demonstrates the advantages that will be introduced to crowded field photometry in close-by galaxies with ELTs.
\end{abstract}

Keywords. Galaxies: star clusters, Hertzsprung-Russell diagram; Stars: imaging, low-mass, brown dwarfs, luminosity function, mass function; Techniques: photometric, telescopes

\section{Introduction}

Observations of low-mass stellar populations and studies of their Initial Mass Function (IMF) are available for our galaxy only, down to the detection limit, which is around $0.1 \mathrm{M}_{\odot}$ or lower (Scalo 1998). Although the IMF appears relatively uniform when averaged over whole clusters or large regions of galaxies (Chabrier 2003), it shows local spatial variations, which could not only reflect sampling limitations in the observations, but may also be the result of physical differences, or even purely statistical in nature (Elmegreen 2004). The galactic IMF is found to be approximately a power law for intermediate- to high-mass stars, but it becomes flat in the low-mass regime (Reid 1998; Kroupa 2002). Observed variations from one region of the Galaxy to the next in the number of low-mass stars and brown dwarfs over intermediate- and high-mass stars affect the corresponding IMF (e.g. Bouvier et al. 1998; Hillenbrand \& Carpenter 2000; Preibisch et al. 2003; Stolte et al. 2005).

Observational constraints for the detection of extra-galactic low-mass stars do not allow similar investigations for other galaxies of the Local Group. The Magellanic Clouds (MCs), our closest undisrupted neighbouring dwarf galaxies, provide a unique sample of young star clusters and stellar associations to be used as laboratories for testing ideas on extra-galactic star formation. A large amount of Hubble Space Telescope (HST) time with the Wide Field Planetary Camera 2 (WFPC2) has been dedicated to young star 

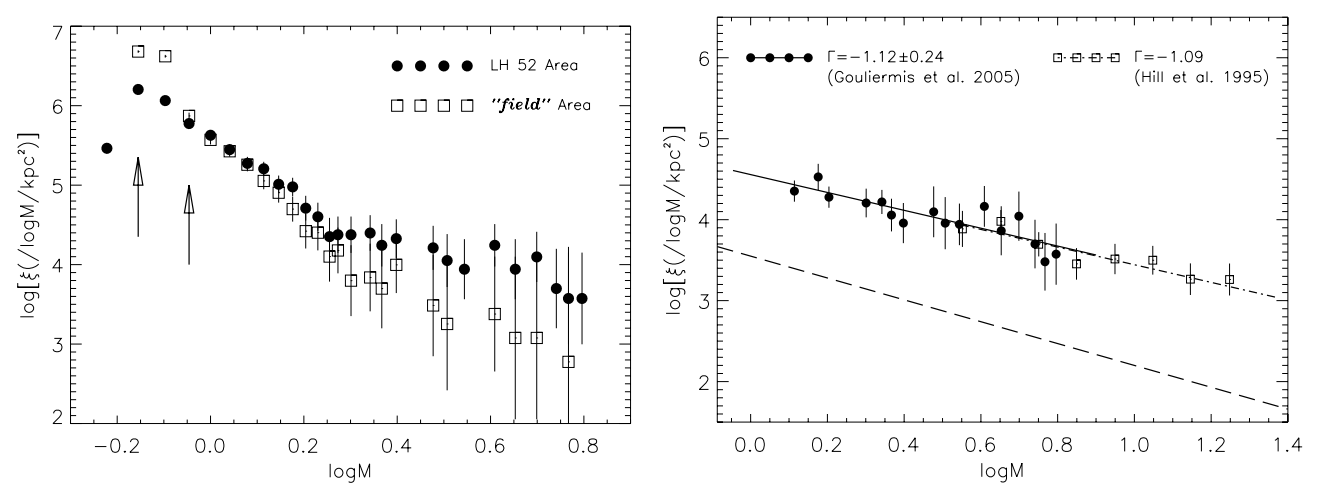

Figure 1. Left: The main sequence present-day mass function of the general field of the LMC in the area of the association LH 52 and of a close-by "empty" background field. The arrows indicate the 50\% completeness level for the LH 52 area (left arrow) and the field (right arrow). Right: The IMF of the association LH 52 down to about $1 M_{\odot}$ after the contribution of the general field has been subtracted. Results form studies on both the high- (Hill et al. 1995) and low-mass (Gouliermis et al. 2005) population of the system are combined for the construction of this IMF through the whole observed mass range up to $\sim 18 \mathrm{M}_{\odot}$.

clusters of the MCs, giving new important results. Still, their central regions, which are characterized by high stellar density, are suffering from crowding, which cannot be bypassed even from space. Therefore, our knowledge is limited to stars with masses down to a few $\mathrm{M}_{\odot}$ in these clusters. Stellar associations, on the other hand, being dynamically unbound, do not suffer so much from crowding, and thus their observations would be more complete towards the low-mass regime. We tested this assumption with WFPC2 observations from the HST Data Archive of the LMC association LH 52 (Gouliermis et al. 2005) and we detected stars with masses down to $\sim 0.7 \mathrm{M}_{\odot}$. The IMF of the association was constructed for stars down to $\sim 1 \mathrm{M}_{\odot}$, due to limited observations of the local background field (Fig. 1). HST/WFPC2 imaging has shown that it is not possible for such low masses to be detected in bound star clusters of the MCs (e.g. de Grijs et al. 2002; Gouliermis et al. 2004). Consequently, if we want to study the sub-solar population in such systems, we should consider the future use of ELTs to solve the crowding problem in dense environments of our close-by galaxies.

\section{Simulated imaging of a LMC cluster with a 100-m class telescope}

The crowding problem in young star clusters of the MCs has been discussed by Olsen et al. (2003), who developed an analytical model to study to what level adaptive optics (AO) corrected ELTs will perform useful photometry. Ardeberg \& Linde (these proceedings) presented their simulations to evaluate the usefulness of ELTs in resolving stellar populations in the Virgo cluster, and Olsen et al. (these proceedings) simulated the performance of ELTs for the study of histories of galaxies at distances of up to $\sim 20 \mathrm{Mpc}$. We perform simulations of imaging with a 100-m telescope, in order to quantify the possibility to separate faint stars at different distances within the Local Group. All these studies deal with the resolving efficiency of ELTs in crowded regions at great distances.

As a template region we use the old LMC cluster NGC 1928 and its observations with the Advanced Camera for Surveys (ACS) on board HST. We performed photometry of the data available in the HST Data Archive, with the package DOLPHOT for ACS, a modified version of HSTPhot (Dolphin 2000). The results for the main part of the cluster 

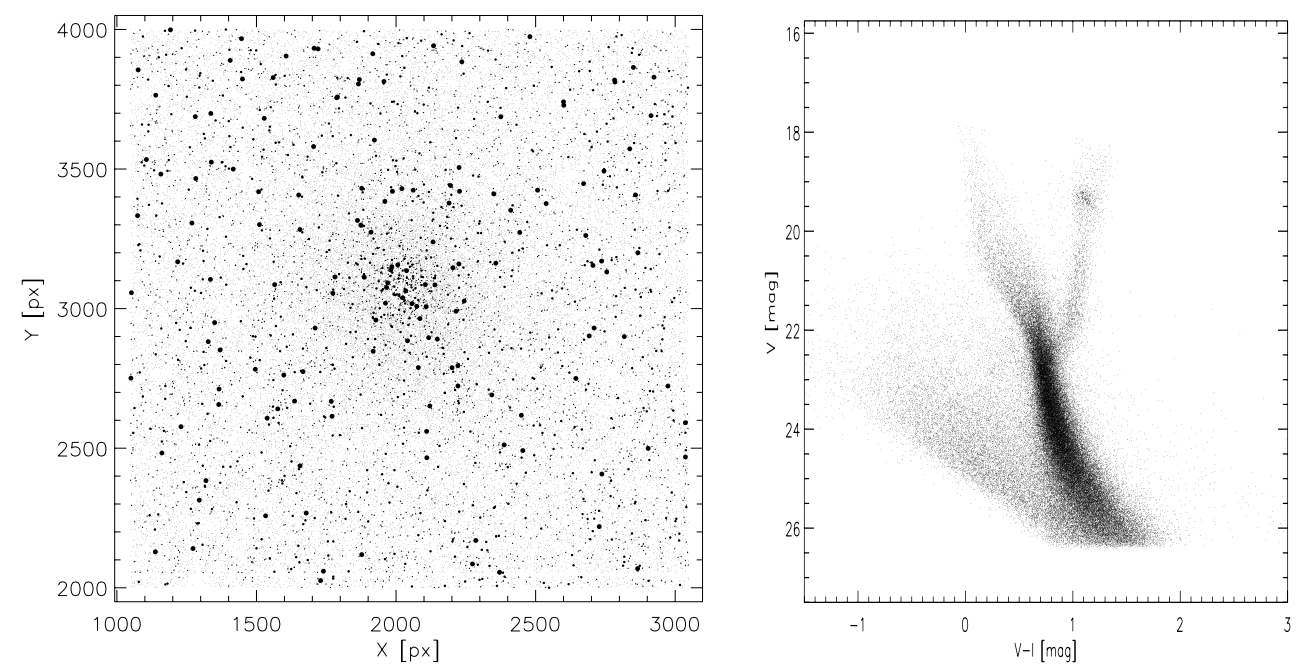

Figure 2. Star chart of the main part of the cluster NGC 1928 observed with HST/ACS and the corresponding CMD of the stars in the map from photometry with DOLPHOT for ACS.

are shown in Fig. 2. We used the star catalog of the whole WFC field-of-view on NGC 1928 to reproduce the stellar distribution of the area in artificial digital images with no PSF effect. Diffraction-limited PSFs with a Strehl ratio of 0.6 simulated by us (Berton et al. these proceedings) for a 100-m class telescope are used for the convolution of the artificial images, in order to simulate observations of the cluster with such a telescope. We show preliminary results in Fig. 3. The high-resolution image of the ELT covers only a narrow field in the center of the cluster with a 100\% efficiency in detecting stars of magnitudes down to the assumed detection limit $(V \sim 26 \mathrm{mag})$. If the cluster is assumed to be located in the Andromeda galaxy, then almost its whole main area is covered by a single telescope pointing, but the star detection efficiency drops as a function of brightness. This efficiency is still above $50 \%$ for stars down to the limit of apparent magnitude $V \sim 18$ mag. This limit is defined by the confusion at the greater distance of M 31 (Fig. 3).

These are preliminary results. More crowded regions, preferably simulated with the Monte Carlo method, including stars down to lower mass limits, and simulations of imaging with ELTs of different aperture are certainly needed. This method would then provide a complete sample of dependencies of separations with distances for regions of different crowding rate and we would define the lower mass that can be reached with several ELTs for different galaxies of the Local Group.

\section{References}

Bouvier, J. et al. 1998, A\&A A 336, 490

Chabrier, G. 2003, PASP 115, 763

de Grijs, R. et al. 2002, MNRAS 337, 597

Dolphin, A.E. 2000, PASP 112, 1383

Elmegreen, B.G. 2004, MNRAS 354, 367

Gouliermis, D. et al. 2004, A\& A 416, 137

Gouliermis, D., Brandner, W. \& Henning, Th. 2005, ApJ 623, 846

Hill, R.S. et al. 1995, ApJ 446, 622 

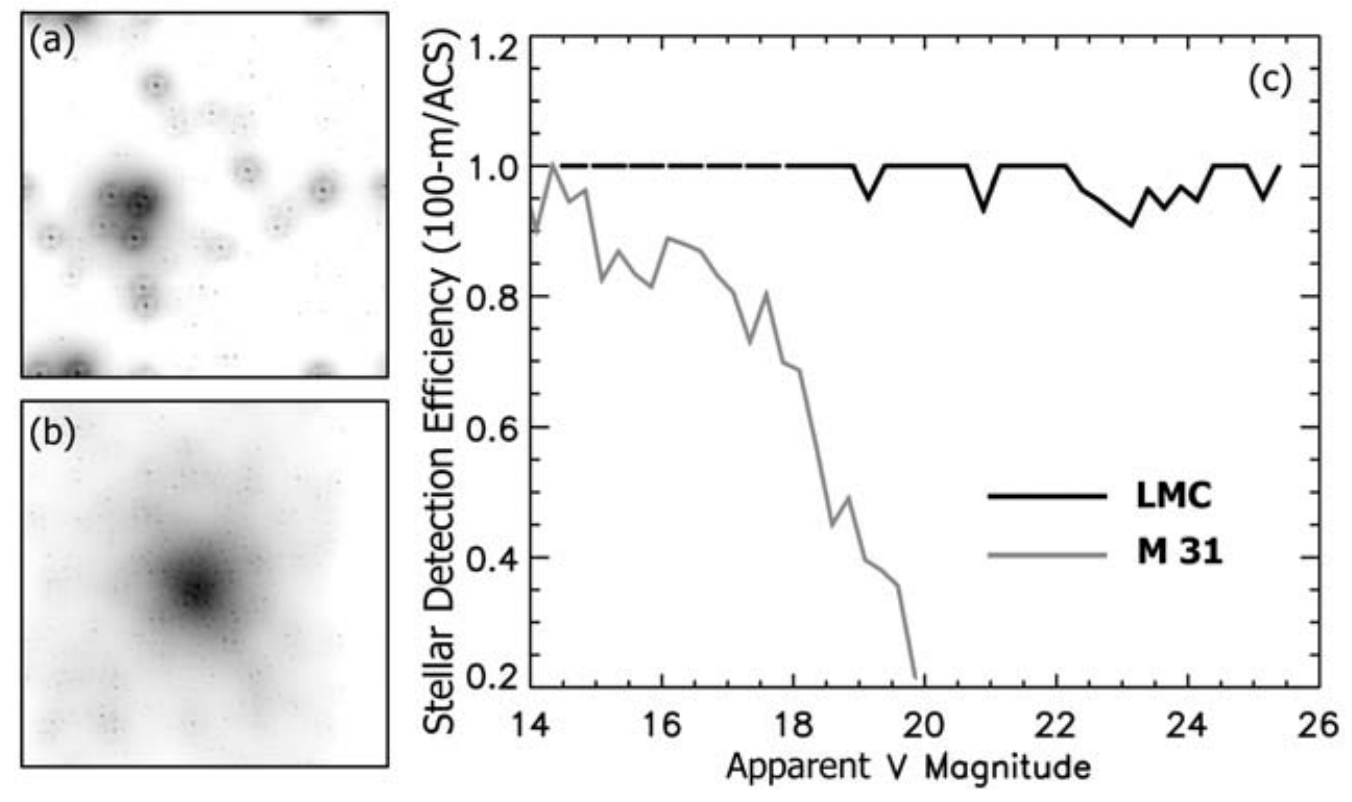

Figure 3. Left: Simulated diffraction-limited images of the central part of the LMC cluster NGC 1928, observed with a 100-m class telescope (a) at its original distance and (b) if it were located in M 31. Right: (c) Star detection efficiency (number of stars recovered after photometry with a 100-m ELT versus number of stars from the input catalog from ACS, at the distance of the LMC (dark line) and M 31 (grey line). The fluctuations represent low-number statistics.

Hillenbrand, L.A. \& Carpenter, J.M. 2000, ApJ 540, 236

Kroupa, P. 2002, Science 295, 82

Olsen, K.A.G., Blum, R.D. \& Rigaut, F. 2003, $A J$ 126, 452

Preibisch, T., Stanke, T. \& Zinnecker, H. 2003, A\& A 409, 147

Reid, N. 1998, in: G. Gilmore \& D. Howell (eds.), The Stellar IMF ASP Conf. Ser. 142, p. 121

Scalo, J. 1998, in: G. Gilmore \& D. Howell (eds.), The Stellar IMF ASP Conf. Ser. 142, p. 201

Stolte, A., Brandner, W., Grebel, E.K., Lenzen, R. \& Lagrange, A.-M. 2005, ApJL 628, L113

\section{Discussion}

LINDE: What is the Strehl ratio for the OWL PSF you used?

Gouliermis: The assumed Strehl ratio for our toy-PSF is 0.6.

Piотто: For the projects you propose, subtraction of field stars is rather important. With the resolution of ELTs in principle this operation can be performed by proper-motion discrimination, at least within the local group. This implies the possibility to measure accurate astrometry (local astrometry) as we did in many cases for MW clusters using HST images: does the PSF variation allow such accurate astrometry.

Gouliermis: It is my impression that astrometry will be advanced by the use of ELTs, taking into account the PSF variation, but in the local universe and especially in the Magellanic Clouds. Our preliminary tests show that even in compact clusters the resolving power of diffraction limited telescopes will allow accurate astrometric measurements. The problem in the case of the Magellanic Clouds is that this will be possible only in very narrow fields of view. So several telescope pointings are needed to cover a respectable area of a cluster. 